\title{
STRUCTURE THEOREMS FOR CERTAIN TOPOLOGICAL RINGS
}

\author{
BY
}

\section{JAMES B. LUCKE AND SETH WARNER}

\begin{abstract}
A Hausdorff topological ring $B$ is called centrally linearly compact if the open left ideals form a fundamental system of neighborhoods of zero and $B$ is a strictly linearly compact module over its center. A topological ring $A$ is called locally centrally linearly compact if it contains an open, centrally linearly compact subring. For example, a totally disconnected (locally) compact ring is (locally) centrally linearly compact, and a Hausdorff finite-dimensional algebra with identity over a local field (a complete topological field whose topology is given by a discrete valuation) is locally centrally linearly compact. Let $A$ be a Hausdorff topological ring with identity such that the connected component $c$ of zero is locally compact, $A / c$ is locally centrally linearly compact, and the center $C$ of $A$ is a topological ring having no proper open ideals. A general structure theorem for $A$ is given that yields, in particular, the following consequences: (1) If the additive order of each element of $A$ is infinite or squarefree, then $A=A_{0} \times D$ where $A_{0}$ is a finite-dimensional real algebra and $D$ is the local direct product of a family $\left(A_{\gamma}\right)$ of topological rings relative to open subrings $\left(B_{\gamma}\right)$, where each $A_{\gamma}$ is the cartesian product of finitely many finite-dimensional algebras over local fields. (2) If $A$ has no nonzero nilpotent ideals, each $A_{y}$ is the cartesian product of finitely many full matrix rings over division rings that are finite dimensional over their centers, which are local fields. (3) If the additive order of each element of $A$ is infinite or squarefree and if $C$ contains an invertible, topologically nilpotent element, then $A$ is the cartesian product of finitely many finite-dimensional algebras over $\mathbf{R}, \mathbf{C}$, or local fields.
\end{abstract}

1. Introduction. Our goal is to identify those properties of a Hausdorff topological ring with identity that allow it to be described as built, in some concrete way, from topological rings that either are finite-dimensional Hausdorff topological algebras over local fields, the real field $\mathbf{R}$, or the complex field $\mathbf{C}$, or are very similar to such algebras. By a local field we mean a topological field whose topology is given by a complete, discrete valuation. Thus a topological field $K$ is locally compact and totally disconnected if and only if $K$ is a local field and the residue field of its valuation ring is finite. Local fields, $\mathbf{R}$, and $\mathbf{C}$ are examples of complete topological fields whose topology is given by a proper absolute value; a finite-dimensional algebra $A$ over such a field admits a unique Hausdorff topology making it a topological vector space, and $A$ is actually a topological algebra for that topology [3, Theorem 2, p. 18; cf.

Received by the editors May 26, 1972.

AMS (MOS) subject classifications (1970). Primary 16A80, 13J10.

Key words and phrases. Topological ring, local field, strictly linear compact ring, centrally linearly compact ring.

Copyright $\odot$ 1974, American Mathematical Society 
Corollary 2, p. 19]. In this introductory section we shall describe the topological rings to be investigated.

We recall that a topological (not necessarily unitary) module is linearly topologized if the open submodules form a fundamental system of neighborhoods of zero. A topological module is linearly compact if it is Hausdorff, linearly topologized, and if every filter base of cosets of submodules has an adherent point. A linearly compact $A$-module $E$ is strictly linearly compact if every continuous epimorphism from $E$ onto any linearly compact $A$-module is open, or equivalently, if $E / U$ is artinian for every open submodule $U$. (We assume familiarity with basic properties of linearly compact and strictly linearly compact modules, discussed in [11] or [4, Exercises 14-22, pp. 108-112].) A topological ring $B$ is (strictly) linearly compact if the left $B$-module $B$ is (strictly) linearly compact.

Definition 1. A Hausdorff topological ring $B$ is centrally linearly compact if the open left ideals of $B$ form a fundamental system of neighborhoods of zero and if $B$ is a strictly linearly compact module over its center. A topological ring $A$ is locally centrally linearly compact if $A$ contains an open subring that is centrally linearly compact.

If $B$ is centrally linearly compact and if $R$ is any subring of $B$ that contains the center $C_{B}$ of $B$, then $B$ is a strictly linearly compact (left) $R$-module, for $B$ is $R$-linearly topologized as the open left ideals form a fundamental system of neighborhoods of zero, and every $R$-submodule is also a $C_{B}$-submodule. In particular, a centrally linearly compact ring is a strictly linearly compact ring. A commutative topological ring is clearly centrally linearly compact if and only if it is strictly linearly compact.

A totally disconnected locally compact ring $A$ is locally centrally linearly compact, for $A$ contains a compact subring $B$ [10, Lemma 4], and the open ideals of $B$ form a fundamental system of neighborhoods of zero [9, Lemma 9]. We shall determine the structure of a Hausdorff topological ring $A$ with identity that has the following three properties: (a) the connected component $c$ of zero is locally compact; (b) $A / c$ is locally centrally linearly compact; (c) the center of $A$ is a topological ring having no proper open ideals. Thus our results may be applied to obtain the structure of a locally compact ring with identity whose center is a topological ring having no proper open ideals.

Our first step is to identify a very special class of centrally linearly compact rings.

Definition 2. A topological ring $B$ is basic if it satisfies the following three properties:

1. The center $C_{B}$ of $B$ is a local, one-dimensional noetherian ring (with identity) that is complete for its natural topology (i.e., the m-adic topology, where 
$m$ is the maximal ideal of $C_{B}$ ), and the prime ideals of the zero ideal of $C_{B}$ are all isolated.

$2^{\circ}$. $B$ is a noetherian $C_{B}$-module, and the given topology of $B$ is its natural topology as a $C_{B}$-module (for which $\left(m^{n} B\right)_{n \geq 0}$ is a fundamental system of neighborhoods of zero).

$3^{\circ}$. Every cancellable element (i.e., non-zero-divisor) of the ring $C_{B}$ is also a cancellable element of $B$.

Condition $3^{\circ}$ implies that the identity element of $C_{B}$ is also the identity element of $B$. By $2^{\circ}$ and [18, Theorem 4, p. 254], the topology induced on $C_{B}$ by the given topology of $B$ is its natural topology. Since $C_{B}$ is complete for that topology, therefore, $B$ is also complete [18, Theorem 5, p. 256]. By [18, pp. 271272], $C_{B}$ is a linearly compact ring. Since $\mathrm{m}^{n} / \mathrm{m}^{n+1}$ is a finitely generated $C_{B}$. module and hence a finite-dimensional $\left(C_{B} / \mathrm{m}\right)$-vector space, $\mathrm{m}^{n} / \mathrm{m}^{n+1}$ is an artinian $C_{B}$-module for all $n \geq 0$, so by induction $C_{B} / m^{k}$ is an artinian $C_{B}{ }^{*}$ module for all $k \geq 1$; thus $C_{B}$ is a strictly linearly compact ring. $A s B$ is a finitely generated $C_{B}$-module, $B$ is, for some $r \geq 1$, the image of the $C_{B}$-module $C_{B}^{r}$ under a continuous homomorphism, and hence $B$ is a strictly linearly compact $C_{B}$-module. In sum, we have proved the following theorem.

Theorem 1. A basic topological ring is centrally linearly compact.

Let $p_{1}, \cdots, p_{r}$ be the prime ideals of the zero ideal of $C_{B}$. By $1^{\circ}$ of Definition 2, $m, p_{1}, \ldots, p_{r}$ are all the prime ideals of $C_{B}$, and $m \neq p_{i}$ for all $i \epsilon$ $[1, r]$. The set of zero-divisors of $B$ belonging to $C_{B}$ is identical with the set of zero-divisors of the ring $C_{B}$ by $3^{\circ}$ of Definition 2, and that set is $\bigcup_{i=1}^{r} p_{i}$ $[17$, Corollary $3, \mathrm{p} .214]$. Therefore, since $\mathrm{m} \notin \bigcup_{i=1}^{r} p_{i}[17, \mathrm{p} .215], m$ contains a cancellable element of $B$. If $c$ is any cancellable element belonging to $m$, then $m$ is the only prime ideal of $C_{B}$ containing $C_{B} c$, so $C_{B} c$ is primary for $m$ and thus $\mathrm{m}^{k} \subseteq C_{B} c$ for some $k \geq 0$.

Let $A$ be the total quotient ring of $B$; thus $A$ is the ring of all fractions $a / c$ where $a \in B$ and $c$ is a cancellable element of $B$ belonging to $C_{B}$ (or equivalently, by $3^{\circ}$ of Definition 2, a cancellable element of $C_{B}$ ). Then $\left(m^{n} B\right)_{n \geq 1}$ is a fundamental system of neighborhoods of zero for a topology $\mathfrak{T}$ on $A$ making $A$ into an additive topological group. Since $B$ is open in $A$ for $\mathfrak{T}$, the multiplicative composition of $A$ is continuous at $(0,0)$, and for each $b \in B$ the functions $x \mapsto b x$ and $x \mapsto x b$ from $A$ into $A$ are continuous at zero. To show that $A$, equipped with topology $\mathcal{T}$, is a topological ring, therefore, it suffices to show that if $c$ is any cancellable element of $B$ belonging to $\mathrm{m}$, then $x \mapsto c^{-1} x$ is continuous at zero, or equivalently, $x \mapsto c x$ is an open mapping. But as noted above, $\mathrm{m}^{k} \subseteq C_{B} c$ for some $k \geq 1$, so $\mathrm{m}^{n+k} B \subseteq c\left(\mathrm{~m}^{n} B\right)$ for all $n \geq 1$; thus 
$x \mapsto c x$ is open. Therefore we have proved the following theorem.

Theorem 2. Let $B$ be a basic topological ring, and let $A$ be the total quotient ring of $B$. Equipped with the topology for which the open neighborboods of zero in $B$ form a fundamental system of neighborboods of zero, $A$ is a topological ring containing $B$ as an open subring; consequently, $A$ is a locally centrally linearly compact ring.

The total quotient ring $A$ of a basic ring $B$, topologized as indicated, is called the topological quotient ring of $B$.

In view of the goal we have set ourselves, we need to define a class of topological rings that contains the class $\mathfrak{Q}_{0}^{\prime}$ of all topological rings that happen to be Hausdorff finite-dimensional topological algebras with identity over local fields but, in some sense, is not much larger than that class. A moment's reflection suggests, however, that $\mathfrak{Q}_{0}^{\prime}$ is not the class we should consider, for by classical Wedderburn theory, a finite-dimensional algebra with identity over a field $K$ is isomorphic to the cartesian product of finitely many finite-dimensional algebras over $K$ whose centers are local subalgebras (a local algebra is an algebra that, regarded as a ring, is a local ring, i.e., is commutative, has an identity, and has only one maximal ideal). Indeed, if $C$ is the center of a finitedimensional algebra $A$ with identity 1 , then $C / \operatorname{Rad}(C)$ is the direct sum of finitely many fields by Wedderburn's theorem; raising idempotents, we obtain orthogonal idempotents $e_{1}, \ldots, e_{n}$ in $C$ whose sum is 1 such that each $C e_{i}$ is a local algebra [8, Proposition 4, p. 54]; then $A$ is the direct sum of the ideals $A e_{1}$, $\ldots, A e_{n}$, and the center of $A e_{i}$ is $C e_{i}$. Therefore, instead of $\mathfrak{C}_{0}^{\prime}$, we shall seek an appropriate extension of the class $\mathfrak{Q}_{0}$ of all cartesian products of finitely many topological rings that happen to be finite-dimensional Hausdorff topological algebras with identity over local fields; each member of $\mathfrak{Q}_{0}$ is thus the cartesian product of finitely many members of the class $\mathfrak{Q}_{0 s}$ of all topological rings that happen to be finite-dimensional Hausdorff topological algebras with identity over local fields, the centers of which are local subalgebras.

We contend that the appropriate extension of $\mathbb{C}_{0}$ is the class $\mathbb{Q}$ of all cartesian products of finitely many topological quotient rings of basic rings. The analogue of $\mathfrak{Q}_{0 s}$ in $\mathbb{Q}$ is the class $\mathbb{Q}_{s}$ of all topological quotient rings of basic rings that are special in the following sense:

Definition 3. A commutative ring with identity is special if every zerodivisor is nilpotent, that is, if ( 0$)$ is a primary ideal. A basic topological ring is special if its center is a special ring.

Our contention is based on the following facts: (1) A topological field is a local field if and only if it is locally strictly linearly compact (i.e., has an open, 
strictly linearly compact subring), or equivalently, if and only if it is the topological quotient field of a basic topological integral domain. (2) More generally, a topological division ring is finite-dimensional over its center and its topology is given by a complete, discrete valuation if and only if it is locally centrally linearly compact, or equivalently, if and only if it is the topological quotient ring of a basic topological ring having no proper zero-divisors. (3) If $A \in \mathfrak{Q}$, then $A$ has many of those properties possessed by all members of $\mathfrak{Q}_{0}$, e.g., $A$ is complete, $A$ is artinian and noetherian over its center $C$, every proper $C$-submodule (in particular, every proper left or right ideal) of $A$ is closed but not open, and every cancellable element of $A$ is invertible. (4) $\mathfrak{Q}_{0 s}$ consists precisely of those members of $\mathbb{Q}_{s}$ that have either zero or prime characteristic. (5) Each member of $\mathbb{Q}$ is the cartesian product of finitely many members of $\mathbb{Q}_{s} \cdot(6) \mathbb{Q}_{0}$ consists precisely of those members of $\mathbb{Q}$ the additive order of each element of which is either infinite or a squarefree integer.

In $\$ 2$ we shall prove (3)-(6). (Statements (1) and (2) have essentially been proved in [16, Theorems 1 and 2 and Lemma 2].) The crucial step in establishing (4) is to show that the center $C$ of the topological quotient ring $A$ of a special basic ring of zero or prime characteristic contains a local "coefficient" subfield, i.e., a subfield that is local for its induced topology and is mapped homeomorphically by the canonical epimorphism onto the residue field of $C$. Essential tools for this step are the theorems of I. S. Cohen on complete local noetherian rings.

The structure of topological rings satisfying the above-listed conditions (a)-(c) is given in $\$ 3$. The need for taking members of $\mathbb{Q}$ rather than $\mathbb{Q}_{s}$ as our basic units, despite ( 5 ), arises from the fact that our structure theorems involve not only the topological quotient rings of basic rings, but also the basic rings themselves. We obtain several generalizations of the structure the orem of Goldman and Sah [7, Theorem 4.1] for locally compact, commutative, semisimple rings with identity that have no proper open ideals. We also characterize those (not necessarily locally compact) topological rings with identity that are cartesian products of finitely many finite-dimensional Hausdorff topological algebras over $\mathbf{R}, \mathbf{C}$, or a local field; this theorem generalizes a structure theorem [13, Theorem 8] for locally compact rings whose center contains an invertible, topologically nilpotent element.

2. Topological quotient rings of basic rings. We shall first establish assertion (3) of $\$ 1$.

Theorem 3. Let $A$ be the topological quotient ring of a basic ring $B$, let $C_{B}$ be the center of $B$, let $m$ be the maximal ideal of $C_{B}$, let $p_{1}, \ldots$, $p_{r}$ be 
the remaining prime ideals (namely, the prime ideals of the zero ideal) of $C_{B}$, and let $C$ be the center of $A$.

1. $C_{B}=C \cap B$, and $C$ (with its induced topology) is the topological quo. tient ring of $C_{B}$.

$2^{\circ}$. The only prime ideals of $C$ are $C p_{1}, \ldots, C p_{r}$ and $C P_{i} \cap C_{B}=p_{i}$ for all $i \in[1, r]$; thus $C$ is a zero-dimensional noetberian ring and bence is an artinian ring.

3.. $A$ is an artinian (noetberian) C-module; in particular, $A$ is a (left or right) artinian (noetherian) ring.

$4^{\circ}$. A is complete.

$5^{\circ}$. Every C.submodule of $A$ is closed (in particular, every left or right ideal of $A$ is closed, and every ideal of the topological ring $C$ is closed).

60. No proper C-submodule of $A$ is open (in particular, no proper left or right ideal of $A$ is open), and no proper ideal of the topological ring $C$ is open.

$7^{\circ}$. Every cancellable element of $A$ is invertible.

Proof. Since $A$ is the total quotient ring of $B$, clearly $C$ is the total quotient ring of $C_{B}$ and $C \cap B=C_{B}$. Therefore $C_{B}$ is open in $C$, and hence the topology induced on $C$ by that of $A$ makes $C$ the topological quotient ring of $C_{B}$, since as we observed after Definition 2, the topology induced on $C_{B}$ by that of $B$ is its natural topology. Now $C=S^{-1} C_{B}$ where $S=C_{B}-U_{i=1}^{r} p_{i}$; hence $C$ is a noetherian ring whose only prime ideals are $C P_{1}, \ldots, C P_{r}$, and $C P_{i} \cap$ $C_{B}=p_{i}$ for all $i \in[1, r][17, p .224]$; therefore each $C p_{i}$ is a maximal ideal. Thus $C$ is an artinian ring [17, Theorem 2, p. 203].

To prove $3^{\circ}$, it suffices by $2^{\circ}$ to show that $A$ is a finitely generated $C$-module. But by definition, $B$ is finitely generated over $C_{B}$, and it is immediate that if $B=C_{B} b_{1}+\cdots+C_{B} b_{n}$, then $A=C b_{1}+\cdots+C b_{n}$. Since $B$ is a strictly linearly compact $C_{B}$-module by Theorem $1, B$ is complete, and hence as $B$ is open in $A$, $A$ is also complete [1, Corollary 2, p. 46]. By [18, Theorem 9, p. 262], every $C_{B}$-submodule of $B$ is closed. If $M$ is a $C$-submodule of $A$, therefore, $M \cap B$ is is closed in $B$, so $M$ is locally closed at zero, and consequently $M$ is closed [1, Proposition 4, p. 18].

Let $c$ be a cancellable element of $C_{B}$ contained in $m$ (i.e., let $c \in \mathfrak{m}-$ $\left.\bigcup_{i=1}^{r} p_{i}\right)$. Then $c$ is invertible in $C$, and $\lim c^{n}=0$. Thus every open neighborhood of zero in the topological ring $C$ contains the invertible element $c^{n}$ for some $n$; hence $C$ is the only open ideal of the topological ring $C$. Let $M$ be an open $C$-submodule of $A$. Given $z \in A$, there exists $n \geq 1$ such that $c^{n} z \in M$ since $\lim c^{n} z=0$, so $z=c^{-n}\left(c^{n} z\right) \in C M \subseteq M$; hence $M=A$.

Finally, let $a$ be a cancellable element of $A$. As $A$ is a left artinian ring, 
there exists $n \geq 0$ such that $A a^{n}=A a^{n+1}$, whence $a^{n}=b a^{n+1}$ for some $b \in A$ and thus $1=b a$; similarly, as $A$ is a right artinian ring, $a$ is right invertible.

Theorem 4. Let $A$ be the topological quotient ring of a commutative, special basic ring $B$, and let $p$ be the radical of $(0)$ in $B$. Then $A$ is a noetherian primary ring whose unique prime ideal is $A$ fo and the field $A / A p$, equipped with its induced topology, is a local field.

Proof. The first assertion follows from $2^{\circ}$ and $3^{\circ}$ of Theorem 3. Since $A p$ is closed but not open by $5^{\circ}$ and $6^{\circ}$ of Theorem $3, A / A \emptyset$ is a Hausdorff, indiscrete topological ring that algebraically is a field. Since $B$ is strictly linearly compact by Theorem 1, its image in $A / A p$ under the canonical epimorphism is an open, strictly linearly compact subring of $A / A \not$. Therefore $A / A p$ is a local field [16, Theorem 1].

Lemma 1. Let $A$ be the topological quotient ring of a commutative, special basic ring $B$. If $B^{\prime}$ is a subring of $A$ that contains $B$ and if $B^{\prime}$ is a finitely generated $B$-module, then $B^{\prime}$ is a special basic ring and $A$ is the topological quotient ring of $B^{\prime}$.

Proof. Since $B$ is open in $B^{\prime}, B^{\prime}$ is a semilocal noetherian ring, and its induced topology is its natural topology [14, Theorem 5]. As $A$ is complete (4\%, Theorem 3) and as $B^{\prime}$ is open and hence closed in $A, B^{\prime}$ is complete. Therefore by a theorem of Chevalley [18, Corollary 2, p. 283], $B^{\prime}$ is the topological direct sum of complete local rings; but as $A$ is local, $A$ has no idempotents other than 0 and 1 ; hence $B^{\prime}$ is a local ring, and $\operatorname{dim} B^{\prime}=1$ [18, Corollary 3, p. 291]. Since $\mathfrak{m}^{\prime} \cap B=\mathfrak{m}$, where $\mathfrak{m}$ and $\mathfrak{m}^{\prime}$ are the maximal ideals of $B$ and $B^{\prime}$ respectively [17, Complement (2), p. 259], $\mathrm{m}^{\prime}$ contains an invertible element of $A$, so $\mathrm{m}^{\prime}$ is not a prime ideal of the zero ideal of $B^{\prime}$. Thus $B^{\prime}$ is a basic ring. Clearly $A$ is the topological quotient ring of $B^{\prime}$. Therefore as $A$ is local, $B^{\prime}$ is special by $2^{\circ}$ of Theorem 3.

Lemma 2. If $A$ is the topological quotient ring of a commutative, special basic ring $B$, then $A$ is the topological quotient ring of a commutative, special basic ring $B^{\prime}$ that contains $B$ and is mapped by the canonical epimorphism onto the valuation ring of the residue field of $A$.

Proof. Let $m$ be the maximal ideal of $B, p$ the radical of $(0)$ in $B$, and $\phi$ the canonical epimorphism from $A$ onto its residue field $A / A \not$. Then $\phi(\mathfrak{m})$ is not the zero ideal of $\phi(B)$, for otherwise $m \subseteq A p \cap B=p$, a contradiction. Thus $\operatorname{dim} \phi(B)=1$, and in particular, $\phi(B)$ is not a field. As $\phi$ is an open mapping, the induced topology of $\phi(B)$ is its natural topology, and $\phi(B)$ is complete since 
it is open and thus closed in $A / A \uparrow$. By $\left[14,1^{\circ}\right.$ of Theorem 6$]$, the integral closure $V$ of $\phi(B)$ in $A / A p$ is a finitely generated $\phi^{\prime}(B)$-module, and $V$ is a local noetherian domain whose induced topology is its natural topology; since $\operatorname{dim} V=\operatorname{dim} \phi(B)=$ 1 [18, Corollary 3, p. 291], $V$ is a discrete valuation ring [18, Corollary 1, p. 42]. Since $V$ is open in $A / A \emptyset$, the topology of $A / A p$ is given by the discrete valuation associated to $V$, so $V$ is the valuation ring of $A / A$.

Let $c_{1}, \ldots, c_{n} \in A$ be such that $V=\phi(B)\left[\phi\left(c_{1}\right), \ldots, \phi\left(c_{n}\right)\right]$, and let $B^{\prime}=$ $B\left[c_{1}, \ldots, c_{n}\right]$. Clearly $\phi\left(B^{\prime}\right)=V$. As $V$ is a finitely generated $\phi(B)$-module, each $\phi\left(c_{k}\right)$ is integral over $\phi(B)$, whence as the kernel $A p$ of $\phi$ is nilpotent, each $c_{k}$ is integral over $B$. Therefore $B^{\prime}$ is a finitely generated $B$-module. The assertion consequently follows from Lemma 1.

If $V$ is a discrete valuation ring, then the discrete topology and the natural topology of $V$ are the only Hausdorff linear topologies on $V$ (i.e., Hausdorff linear topologies on the $V$-module $V$ ), for if $c$ generates the maximal ideal of $V,\left(V c^{n}\right)_{n \geq 0}$ is a sequence consisting of all nonzero ideals of $V$. Therefore any algebraic isomorphism from one Hausdorff linearly topologized discrete valuation ring onto another is a topological isomorphism if neither topology is discrete.

We recall that a subfield $K$ of a local noetherian equicharacteristic ring $A$ is a coefficient field if $K$ is mapped onto the residue field of $A$ by the canonical epimorphism. Moreover, a subring $C$ of a complete local noetherian ring $A$ of characteristic zero whose residue field has prime characteristic $p$ is a coefficient ring if $C$ is a complete, discrete valuation ring whose maximal ideal is generated by $p .1$, if the induced topology of $C$ is its natural topology, and if $C$ is mapped onto the residue field of $A$ by the canonical epimorphism.

Theorem 5. If $A$ is the topological quotient ring of a commutative, special basic ring and if the characteristic of $A$ is either zero or a prime, then there is a subfield $K$ of $A$ that is a local field for its induced topology and is mapped bomeomorpbically onto the residue field of $A$ by the canonical epimorphism.

Proof. By Lemma 2, $A$ is the topological quotient ring of a special basic ring $B$ that is mapped onto the valuation ring $V$ of the residue field of $A$ by the canonical epimorphism $\phi$. Let $m$ be the maximal ideal of $B, b$ the other prime ideal of $B$; then $A / A p$ is the residue field of $A$. The restriction $\phi_{B}$ of $\phi$ to $B$ is thus an epimorphism from $B$ onto $V$, and it induces an isomorphism $\boldsymbol{\phi}_{B}$ from the residue field $B / m$ of $B$ onto the residue field $V / \phi(m)$ of $V$ such that $\bar{\phi}_{B} \circ \sigma=\rho \circ \phi_{B}$, where $\sigma$ and $\rho$ are respectively the canonical epimorphisms from $B$ onto $B / \mathrm{m}$ and from $V$ onto $V / \phi(\mathrm{m})$. Since the maximal ideal $A p$ of $A$ is nilpotent, the characteristic of $A / A p$ is zero if the characteristic of $A$ is. Therefore $B$ and $V$ have the same characteristic. 
Case 1. $V$ is equicharacteristic (and hence also $B$ is equicharacteristic). By Cohen's theorem [6, Theorem 9], [12, (31.1)], $B$ contains a subfield $k$ mapped onto $B / \mathfrak{m}$ by $\sigma$. Thus $\bar{\phi}_{B} \circ \sigma=\rho \circ \phi_{B}$ maps $k$ isomorphically onto the residue field $V / \phi(m)$ of $V$, so the restriction $\phi_{k}$ of $\phi$ to $k$ is an isomorphism from $k$ onto a coefficient field of $V$. Since $V$ is a complete, regular, local, onedimensional ring, there is a topological isomorphism $F$ from the ring $k[[X]]$ of formal power series over $k$, equipped with its natural (i.e., $X$-adic) topology, onto $V$ that extends $\phi_{k}[6$, Theorem 15], [12, (31.12)]. Let $c \in B$ be such that $F(X)=$ $\phi(c)$. Then $c \in \mathrm{m}$, so as $B$ is complete for the m-adic topology, $\left(a_{n} c^{n}\right)_{n \geq 0}$ is summable for any sequence $\left(a_{n}\right)_{n \geq 0}$ in $k$; thus $S: \Sigma_{k=0}^{\infty} \alpha_{k} X^{k} \mapsto \Sigma_{k=0}^{\infty} \alpha_{k} c^{k}$ is a continuous epimorphism from $k[[X]]$ onto a $k$-subalgebra $k[[c]]$ of $B$. Since $F, S$, and the restriction $\phi_{k}[[c]]$ of $\phi$ to $k[[c]]$ are continuous and since $\phi_{k}[[c]] \circ S$ and $F$ agree on $k$ and at $X$, we conclude that $\phi_{k}[[c]] \circ S=F$. Therefore as $F$ is an isomorphism, $S$ is injective and $\phi_{k}[[c]]$ is surjective, whence $\phi_{k}[[c]]=$ $F \circ S^{-1}$ is an isomorphism from $k[[c]]$ onto $V$. The induced topologies of both $k[[c]]$ and $V$ are Hausdorff, linear, and not discrete, so $\phi_{k}[[c]]$ is a topological isomorphism from $k[[c]]$ onto $V$. Moreover, as $\phi_{k}[[c]]$ is injective, $k[[c]] \cap A p=$ (0), so as $A$ is local, $k[[c]]$ has a quotient field $K$ in $A$. Since $\phi(k[[c]])=V$, clearly $\phi(K)=A / A p$. To show that the restriction of $\phi$ to $K$ is a topological isomorphism from $K$ onto the local field $A / A \uparrow$, therefore, it suffices to show that $k[[c]]$ is open in $K$, Since $k[[c]]$ is a discrete valuation ring, $k[[c]]$ is maximal in the set of proper subrings of $K$ [5, Proposition 6, p. 115]; but $B \cap K$ is a proper subring of $K$ containing $k[[c]]$ since $c$ is not invertible in $B$; therefore $k[[c]]=B \cap K$, so $k[[c]]$ is open in $K$.

Case 2. The characteristic of $V$ is zero and the characteristic of $V / \phi(m)$ is a prime $p$. By Cohen's theorem, $B$ contains a coefficient ring $C[6$, Theorem $11],[12,(31.1)]$. Let $C_{0}=\phi(C)$. Since $A \emptyset$ is nilpotent, $C \cap A \not=(0)$, so the restriction $\phi_{c}$ of $\phi$ to $C$ is an isomorphism from $C$ onto $C_{0}$. Moreover, $C_{0}$ is not discrete, since the $p$-fold of the identity belongs to the maximal ideal of $V$ (and hence is a topological nilpotent) and to $C_{0}$. Therefore $\phi_{C}$ is a topological isomorphism from $C$ onto $C_{0}$; in particular, $C_{0}$ is a complete, discrete valuation ring and its induced topology is its natural topology. Consequently, $C_{0}$ is a coefficient ring of $V$, for $V / \phi(m)=\rho(V)=\rho\left(\phi_{B}(B)\right)=\bar{\phi}_{B}(\sigma(B))=\bar{\phi}_{B}(\sigma(C))=$ $\rho\left(\phi_{B}(C)\right)=\rho\left(C_{0}\right)$.

Since $C \cap A p=(0), C$ has a quotient field $L$ in $A$; let $L_{0}$ be the quotient field of $C_{0}$ in $A / A p$. As in Case 1 , since $C$ and $C_{0}$ are maximal in the set of proper subrings of $L$ and $L_{0}$ respectively, $B \cap L=C$ and $V \cap L_{0}=C_{0}$, so $C$ and $C_{0}$ are open in $L$ and $L_{0}$ respectively; therefore $L$ and $L_{0}$ are local fields and the restriction $\phi_{L}$ of $\phi$ to $L$ is a topological isomorphism from $L$ onto $L_{0}$. 
Since $V$ is a complete, local, one-dimensional noetherian domain, $V$ is a finitely generated $C_{0}$-module by a theorem of Cohen $\left[6\right.$, Theorem 16], $\left[12,(31.0)\right.$. Let $\beta_{1}, \cdots, \beta_{n} \epsilon$ $V$ be such that $V=C_{0} \beta_{1}+\cdots+C_{0} \beta_{n}$. Then $A / A p=L_{0} \beta_{1}+\cdots+L_{0} \beta_{n}$; indeed, if $a \in A$, then $p^{m} a \in B$ for some $m \geq 0$ since $\lim p^{n} a=0$, so $\phi\left(p^{m} a\right)=$ $\gamma_{1} \beta_{1}+\cdots+\gamma_{n} \beta_{n}$ for suitable $\gamma_{i} \in C_{0}$, whence $\phi(a)=\left(p^{-m} \gamma_{1}\right) \beta_{1}+\cdots+$ $\left(p^{-m} \gamma_{n}\right) \beta_{n} \in L_{0} \beta_{1}+\cdots+L_{0} \beta_{n}$. Let $K$ be maximal in the set of all subfields of $A$ containing $L$. Since the characteristic of $A$ is zero, the coefficient fields of $A$ are precisely its maximal subfields [6, proof of Theorem 9]; thus $\phi(K)=$ $A / A \wp$. Let $b_{i} \in K$ be such that $\phi\left(b_{i}\right)=\beta_{i}$. Then $K=L b_{1}+\cdots+L b_{n}$, for if $z \in K$, then $\phi(z)=\phi\left(c_{1}\right) \beta_{1}+\cdots+\phi\left(c_{n}\right) \beta_{n}$ for suitable $c_{i} \in L$, and therefore $z-\sum_{i=1}^{n} c_{i} b_{i} \in K \cap A p=(0)$. The induced topology on $K$ thus makes $K$ a finitedimensional Hausdorff vector space over the local field $L$; as $\phi_{L}$ is a topological isomorphism from $L$ onto $L_{0}$, therefore, it follows from [3, Corollary 2, p. 19] that the restriction of $\phi$ to $K$ is a topological isomorphism from $K$ onto $A / A p$.

A Coben algebra is a local algebra whose maximal ideal has codimension one.

Theorem 6. Let $A$ be a Hausdorff topological ring with identity. The following statements are equivalent:

$1^{\circ}$. $A$ is the topological quotient ring of a special basic ring, and the characteristic of $A$ is either zero or a prime.

2. $A$ is a finite-dimensional topological algebra over a local field $K$, and the center of $A$ is a Coben subalgebra.

30. $A$ is a finite-dimensional topological algebra over a local field $K$, and the center of $A$ is a local subalgebra.

Proof. Let $C$ be the center of $A$. Assume $1^{\circ}$. By $1^{\circ}$ of Theorem 3 and by Theorem 5, $C$ contains a subfield $K$ that is local for its induced topology and is mapped homeomorphically onto the residue field of $C$ by the canonical epimorphism. Thus $C$ is a Cohen algebra over $K$. Since the maximal ideal of $C$ is finitely generated and nilpotent (Theorem 4 ), $C$ is finitely generated over $K$. Since $A$ is a finitely generated $C$-module ( $3^{\circ}$, Theorem 3 ), therefore, $A$ is a finite-dimensional $K$-algebra. Thus $2^{\circ}$ holds.

Assume $3^{\circ}$, and let $V$ be the valuation ring of $K$. As in the proof of $[16$, Theorem 2], there is a basis $f_{1}=1, f_{2}, \ldots, f_{n}$ of the $K$-vector space $A$ such that $B=V f_{1}+\cdots+V f_{n}$ is an open subring, the open ideals of $B$ form a fundamental system of neighborhoods of zero, and $B$ is a strictly linearly compact $V$-module. The center $C_{B}$ of $B$ is contained in $C$; indeed, if $x \in C_{B}$ and if $y \in A$, there exists a nonzero scalar $\lambda \in V$ such that $\lambda y \in B$ since $B$ is open, so $\lambda x y=x(\lambda y)=(\lambda y) x=\lambda y x$, and therefore $x y=y x$. Consequently, $C_{B}=C \cap B$. 
As $V$ is noetherian, $B$ is a noetherian $V$-module, so $C_{B}$ is also a noetherian $V$-module and hence a noetherian ring; moreover, as $C_{B}$ is closed in $B, C_{B}$ is a strictly linearly compact $V$-module and hence is a strictly linearly compact ring. Therefore by [4, Exercise 5(a), p. 117], $C_{B}$ is a complete, semilocal noetherian ring, and its induced topology is its natural topology. But since $C$ is local, $C$ has no idempotents other than 0 and 1 , so $C_{B}$ is a local ring by [18, Corollary 2, p. 283]. As $C_{B}$ is finitely generated and thus integral over $V, \operatorname{dim} C_{B}=\operatorname{dim} V$ $=1$, and the maximal ideal $\mathrm{m}$ of $C_{B}$ intersects $V$ in its maximal ideal; as each nonzero element of $V$ is invertible in $A$, therefore, $m \cap V$ contains invertible elements of $A$ and hence cancellable elements of $C_{B}$, so $\mathrm{m}$ is not a prime ideal of $(0)$ in $C_{B^{*}}$. Thus $1^{\circ}$ of Definition 2 holds for $B$.

Since $B$ is a noetherian $V$-module, $B$ is also a noetherian $C_{B}$-module. By $\left[15\right.$, Theorem $13,2^{\circ}$ and $\left.7^{\circ}\right]$, the topology of $B$ is stronger than the $m$-adic topol$0 \mathrm{gy}$, which is Hausdorff $[17$, p. 253]; since the topology of $B$ is strictly linearly compact and therefore a minimal Hausdorff linear topology, it consequently is the natural $\mathrm{m}$-adic topology. Thus $2^{\circ}$ of Definition 2 holds for $B$.

Now $A$ is contained in the total quotient ring of $B$, for if $z \in A$, there is a nonzero scalar $\lambda \in V$ such that $\lambda z \in B$ as $B$ is open, whence $z=\lambda^{-1}(\lambda z)$ belongs to the total quotient ring of $B$. The same argument establishes that $C$ is contained in the total quotient ring of $C_{B}=C \cap B$. Therefore if $c$ is a cancellable element of $C_{B}$, then $c$ is cancellable in $C$, so $c$ is invertible in $C$ (and also in $A$ ) as $C$ is a finite-dimensional algebra over a field, and in particular, $c$ is cancellable in $B$. Thus $A$ is the total quotient ring of $B$, and $3^{\circ}$ of Definition 2 holds for $B$. Moreover, the local ring $C$ is also the topological quotient ring of $C_{B}$, so $C_{B}$ is special by $2^{\circ}$ of Theorem 3 .

Theorem 7. If $A$ is the topological quotient ring of a basic ring $B$, then $A$ is the topological direct sum of finitely many ideals, each the topological quotient ring of a special basic ring.

Proof. Let $C$ be the center of $A, C_{B}$ the center of $B$. By $3^{\circ}$ of Theorem 3 applied to $C$ and by [17, Theorem 3, p. 205], there exist orthogonal idempotents $e_{1}, \ldots, e_{r}$ in $C$ such that $\Sigma_{i=1}^{r} e_{i}=1$ and each $C e_{k}$ is a noetherian primary ring. Thus $A$ is the topological direct sum of the ideals $A e_{1}, \ldots, A e_{r}$, since the associated projections $p_{k}: x \mapsto x e_{k}$ are continuous (and open). We shall show that $B e_{k}$ is a special basic ring and that $A e_{k}$ is the topological quotient ring of $B e_{k}$.

Let $m$ be the maximal ideal of $C_{B}$. The restriction of $p_{k}$ to $C_{B}$ is an epimorphism from $C_{B}$ onto $C_{B} e_{k}$ that clearly takes cancellable elements into cancellable elements; hence $C_{B} e_{k}$ is a local noetherian ring, $\operatorname{dim} C_{B} e_{k} \leq 1$, 
and the maximal ideal me $e_{k}$ of $C_{B}{ }^{e}{ }_{k}$ contains cancellable elements and hence is not a prime ideal of the zero ideal of $C_{B} e_{k}$; therefore $\operatorname{dim} C_{B} e_{k}=1$ and the prime ideals of the zero ideal of $C_{B} e_{k}$ are all isolated, since $e_{k}$ is not one of them. Since $p_{k}$ is continuous and ope $\mathrm{n}, B e_{k}$ is open in $A e_{k}$ and $\left(m^{n} B e_{k}\right){ }_{n \geq 1}$ is a fundamental system of neighborhoods of zero in $A e_{k}$. But clearly $\mathfrak{m}^{n} B e_{k}=\mathfrak{m}^{n} e_{k} B e_{k}=$ $\left(m e_{k}\right)^{n} B e_{k}$, so the topology of the $\left(C_{B} e_{k}\right)$-module $B e_{k}$ is its (me $\left.e_{k}\right)^{k}$-adic topology. As $A$ is complete, so is $A e_{k}$, and therefore also the open subring $B e_{k}$ of $A e_{k}$ is complete. Moreover, $B e_{k}$ is a finitely generated $\left(C_{B} e_{k}\right)$-module since $B$ is a finitely generated $C_{B}$-module. Therefore the topology induced on any $\left(C_{B} e_{k}\right)$ submodule of $B e_{k}$ is its (me ${ }_{k}$ )-adic topology [18, Theorem 4, p. 254], and the submodule is closed and he nce complete for that topology [18, Theorem 9, p. 262]. In particular, the topology induced on $C_{B} e_{k}$ is its natural (me $e_{k}$ )-adic topology, and $C_{B} e_{k}$ is complete for that topology. Therefore $C_{B} e_{k}$ is a commutative basic ring.

We observed above that if $y$ is a cancellable element of $C_{B}$, then $y e_{k}$ is cancellable in $C_{B} e_{k}$; therefore as $C$ is the total quotient ring of $C_{B}=C \cap B$, $C e_{k}$ is contained in the total quotient ring of $C_{B} e_{k}$. To show that $C e_{k}$ is the total quotient ring of $C_{B} e_{k}$, it consequently suffices to show that a cancellable element $d_{k}$ of $C_{B} e_{k}$ is invertible in $C e_{k}$. Let

$$
d=d_{k}+\sum_{j \neq k} e_{j} \in C
$$

Suppose that $x d=0$, where $x \in C_{B}$. Then as $x d=x d_{k}+\Sigma_{j \neq k} x e_{j}$, we conclude that $x e_{j}=0$ for all $j \neq k$ and $x d_{k}=x d_{k} e_{k}=0$ since $C$ is the direct sum of $C e_{1}, \ldots, C e_{r}$. But then $x e_{k}=0$ since $x e_{k} d_{k}=x d_{k}=0$ and since $d_{k}$ is cancellable in $C_{B} e_{k}$, so consequently $x=0$. Thus $x d \neq 0$ for all nonzero elements $x$ of $C_{B}$; hence $y d \neq 0$ for all nonzero elements $y$ of the total quotient ring $C$ of $C_{B}$; therefore $d$ is a cancellable and hence invertible element of $C$, so $d_{k}=d e{ }_{k}$ is an invertible element of $\mathrm{Ce}_{k} \cdot$. Consequently, $\mathrm{Ce}_{k}$ is the total quotient ring of $C_{B} e_{k}$. Since the restriction of $p_{k}$ to $C$ is the projection onto $C e_{k}$, an open continuous epimorphism, $C_{B} e_{k}$ is open in $C e_{k}$. Therefore $C e_{k}$ is the topological quotient ring of the basic ring $C_{B} e_{k}$. But as $C e_{k}$ has only one prime ideal, $C_{B} e_{k}$ is special by $2^{\circ}$ of Theorem 3 , applied to $C e_{k}$ and $C_{B} e_{k} \cdot$

The center $C_{k}$ of $B e_{k}$ clearly contains $C_{B} e_{k}$ and hence is a $\left(C_{B} e_{k}\right)$ submodule of $B e_{k^{*}}$ As $B e_{k}$ is a noetherian $\left(C_{B} e_{k}\right)$-module, therefore, so is $C_{k}$. Consequently by Lemma $1, C_{k}$ is a special basic ring for its induced topology. But also, as we saw earlier, the induced topology of the $\left(C_{B} e_{k}\right)$-module $C_{k}$ is its (me $e_{k}$ )-adic topology. Hence if $n_{k}$ is the maximal ideal of $C_{k}$, there exists $s \geq 1$ such that $n_{k}^{s} \subseteq$ me ${ }_{k} C_{k} \subseteq n_{k}$, whence $n_{k}^{s} B e_{k} \subseteq m B e_{k} \subseteq n_{k} B e_{k}$; consequently 
$n_{k}^{s t} B_{k} \subseteq \mathfrak{m}^{t} B e_{k} \subseteq n_{k}^{t} B e_{k}$ for all $t \geq 1$, so the topology of the $C_{k}$-module $B e_{k}$ is its $n_{k}$-adic topology. Thus $1^{\circ}$ and $2^{\circ}$ of Definition 2 hold for $B e_{k}$.

To show that $C_{k} \subseteq C$, it suffices to show that if $x \in C_{k}$ and if $y \in B$, then $x y=y x$; but $x y=\left(x e_{k}\right) y=x\left(y e_{k}\right)=\left(y e_{k}\right) x=y x$. Hence $C_{k}=C_{k} e_{k} \subseteq C e_{k}$. If $y$ is a cancellable element of $C_{B}$, then as we have seen, $y e_{k}$ is cancellable in $C_{B} e_{k}$, thus invertible in the total quotient ring $C e_{k}$ of $C_{B} e_{k}$ and hence in $A e_{k}$, and in particular cancellable in $C_{k}$. Therefore $A e_{k}$ is contained in the total quotient ring of $B e_{k}$. To complete the proof that $3^{\circ}$ of Definition 2 holds for $B e_{k}$ and that $A e_{k}$ is the topological quotient ring of $B e_{k}$, it therefore suffices to prove that every cancellable element $g$ of $C_{k}$ is invertible in $A e_{k}$. Let $c_{k}$ be a cancellable element of $C_{B} e_{k}$ belonging to $e_{k}$; then as $g \in C e_{k}$, as $\lim c_{k}^{m} g=0$, and as $C_{B} e_{k}$ is open in $C e_{k}$, there exists $m \geq 1$ such that $c_{k}^{m} g \in C_{B} e_{k}$. Thus $c_{k}^{m} g$ is a cancellable element of $C_{B} e_{k}$, so as we proved earlier, $c_{k}^{m} g$ is invertible in $C e_{k}$, whence $g$ is invertible in $C e_{k}$ and hence also in $A e_{k}$.

The characteristic of a local ring $C$ is either zero or a power of a prime. Indeed, if the characteristic of $C$ were $r s$ where $r$ and $s$ are relatively prime, then $r .1$ and $s .1$ would be zero-divisors and hence would belong to the maximal ideal of $C$, so the identity element of $C$ would also belong to its maximal ideal since there exist integers $m$ and $n$ such that $1=m r+n s$. Consequently, from Theorems 6 and 7 we obtain the following theorem.

Theorem 8. Let $A$ be a topological ring. The following statements are equivalent:

1. A is the topological direct sum of finitely many ideals, each a finitedimensional Hausdorff topological algebra with identity over a local field.

$2^{\circ}$. A is the topological direct sum of finitely many ideals, each the topological quotient ring of a basic ring, and the additive order of eacb element of $A$ is either infinite or a squarefree integer.

$3^{\circ}$. A is the topological direct sum of finitely many ideals, each a finitedimensional Hausdorff topological algebra with identity over a local field whose center is a Coben subalgebra.

3. The structure theorems. To identify those locally centrally linearly compact rings that are topological quotient rings of basic rings, we shall need the following lemma:

Lemma 3. If $B$ is an open subring of a topological ring $A$ with identity, and if the center $C$ of $A$ is a topological ring baving no proper open ideals, then the center $C_{B}$ of $B$ is $B \cap C$.

Proof. Clearly $B \cap C \subseteq C_{B}$. To show that $C_{B} \subseteq B \cap C$, it suffices to prove 
that if $c \in C_{B}$ and if $a \in A$, then $a c=c a$. Since $B$ is open, $V=\{x \in A: x a \in B\}$ is a neighborhood of zero in $A$. Let $W$ be the annihilator in $C$ of ac-ca. Then $W$ contains $V \cap C$ and hence is an open ideal of $C$, for if $x \in V \cap C$, then $(x a) c$ $=c(x a)=(c x) a=(x c) a$ since $x a \in B, c \in C_{B}$, and $x \in C$. By hypothesis, therefore, $W=C$, so $1 \in W$, and consequently $c a=a c$.

Theorem 9. Let $A$ be a Hausdorff topological ring with identity, let $C$ be the center of $A$, and let $B$ be an open subring of $A$ that contains 1 . Then $B$ is a basic topological ring and $A$ is the topological quotient ring of $B$ if and only if $C$ is a topological ring that has no proper open ideals and $B$ is a centrally linearly compact subring whose center $C_{B}$ is a local ring.

Proof. The condition is necessary by Theorem $1,6^{\circ}$ of Theorem 3, and Definition 2.

Sufficiency. By Lemma 3, $C_{B}=B \cap C_{\text {; }}$ in particular, $C_{B}$ is an open subring of $C$. Let $m$ be the maximal ideal of $C_{B}$. (a) $C_{B}$ is a strictly linearly compact ring, and $m$ is open in $C_{B}$. Indeed, $C_{B}$ is a closed submodule of the $C_{B}$-module $B$ and hence is a strictly linearly compact ring. As the open ideals of $C_{B}$ form a fundamental system of neighborhoods of zero for its induced Hausdorff topology, $m$ is open in $C_{B}$.

(b) $m$ is a finitely generated ideal of $C_{B}$. As $m$ is open in $C_{B}$ and hence in $C, C \mathrm{~m}$ is an open ideal of $C$, so $C \mathrm{~m}=C$, and therefore there exist $c_{1}, \ldots$, $c_{n} \in C$ and $b_{1}, \ldots, b_{n} \in m$ such that $c_{1} b_{1}+\cdots+c_{n} b_{n}=1$. Let $b$ be the ideal $C_{B} b_{1}+\cdots+C_{B} b_{n}$ of $C_{B}$; then $b$ is open, for if $b=\left\{x \in B: x c_{i} \in B, 1 \leq i \leq n\right\}$, then $b$ is an open left ideal of $B$, so $D \cap C_{B}$ is an open ideal of $C_{B}$ contained in $b$ (for if $x \in \delta \cap C_{B}$, then $x c_{i} \in C_{B}$ for each $i \in[1, n]$, whence $x=\left(x c_{1}\right) b_{1}+$ $\left.\cdots+\left(x c_{n}\right) b_{n} \in \mathfrak{b}\right)$. As $C_{B}$ is strictly linearly compact, $C_{B} / \mathfrak{b}$ is an artinian $C_{B}$-module and hence a commutative artinian ring. Consequently, $C_{B} / \mathfrak{b}$ is noetherian, so $m / \mathfrak{b}$ is finitely generated, i.e., there exist $m_{1}, \ldots, m_{r} \in m$ such that $m=C_{B} m_{1}+\cdots+C_{B} m_{r}+\mathfrak{b}$. As $b$ is finitely generated, therefore, so is $m$.

(c) $C_{B}$ is a complete local noetherian ring whose topology is its natural topology. If $D$ is an open ideal of $C_{B}$, then $\overline{\mathrm{m}^{n}} \subseteq 0$ for some $n \geq 1$; indeed, as $C_{B}$ is strictly linearly compact, $C_{B} / D$ is an artinian $C_{B}$-module and hence is a local artinian ring; the maximal ideal $\mathrm{m} / D$ of $C_{B} / \mathrm{D}$ is therefore nilpotent, so $\mathrm{m}^{n} \subseteq 0$ for some $n$, whence $\overline{\mathfrak{m}^{n}} \subseteq D$. Consequently, the induced topology of $C_{B}$ is weaker than its natural topology, and $\bigcap_{n=1}^{\infty} \overline{\mathrm{m}^{n}}=(0)$. The assertion therefore follows from (a), (b), and [15, Theorem 12].

(d) $C$ is a noetherian ring all of whose ideals are closed. Let $a$ be an ideal of $C$. By (c) and [18, Theorem 9, p. 262], a $\cap C_{B}$ is closed in $C_{B} \cdot A s C_{B}$ is open in $C$, therefore, $a$ is locally closed at zero and hence is a closed ideal 
[1, Proposition 4, p. 18]. Moreover, as $C$ has no proper open ideals, $C\left(a \cap C_{B}\right)$ $=a\left[7\right.$, Proposition 1.3]. By (c), there exist $x_{1}, \ldots, x_{n} \in a \cap C_{B}$ such that $a \cap C_{B}=C_{B} x_{1}+\cdots+C_{B} x_{n}$, whence $a=C\left(a \cap C_{B}\right)=C x_{1}+\cdots+C x_{n}$.

(e) $C$ is an artinian ring. By (d) and [17, Theorem 2, p. 203], it suffices to show that a prime ideal $\hat{f}$ of $C$ is maximal. Let $\phi$ be the canonical epimorphism from $C$ onto $C / p$, a Hausdorff integral domain by $(d)$. Then $\phi\left(C_{B}\right)$ is a strictly linearly compact $C_{B}$-module and hence is clearly a strictly linearly compact ring; also $\phi\left(C_{B}\right)$ is local and noetherian by (c); therefore the topology of $\phi\left(C_{B}\right)$ is its natural topology [4, Exercise $5(a)$, p. 117], and $\phi\left(C_{B}\right)$ is consequently complete for that topology. In particular, $\phi\left(C_{B}\right)$ is not a field, for in the contrary case its topology would be discrete, so $\cap \cap C_{B}$ would be open in $C_{B}$ and hence in $C$, since the canonical isomorphism from $C_{B} /\left(p \cap C_{B}\right)$ onto $\phi\left(C_{B}\right)$ is continuous, and therefore $p$ would be open in $C$, in contradiction to our hypothesis. Moreover, $C / p$ has no proper open ideals, for if $D$ were a proper open ideal of $C / p, \phi^{-1}(D)$ would be a proper open ideal of $C$. Thus by $1^{\circ}$ of [14, Theorem $6], C / p$ is not integral over $\phi\left(C_{B}\right)$, so by $2^{\circ}$ of $[14$, Theorem 6$], C / F$ is a field, i.e., $p$ is maximal.

(f) There is an invertible element $c$ of $C$ belonging to $m$. By (e) and [17, Theorem 3, p. 205], $C$ has only finitely many maximal ideals. Since each maximal ideal of $C$ is closed but not open by (d), the union of the maximal ideals of $C$ is a closed set having no interior point [2, Proposition 1, p. 107], so the set $G$ of invertible elements of $C$ is open and dense. Thus as $m$ is open, $G \cap m \neq \varnothing$.

(g) $B$ satisfies $1^{\circ}$ of Definition 2, i.e., $C_{B}$ is a basic ring. Clearly $C_{B} c$ $\subseteq \mathrm{m}$. Since $c$ is an invertible element of $C$ belonging to $\mathrm{m}$ and since $C_{B}$ is open in $C, C_{B} c$ is an open ideal of $C_{B}$, hence $C_{B} c$ contains $\mathrm{m}^{n}$ for some $n \geq 1$ by (c), so $C_{B} c$ is primary for $m$ and thus $\operatorname{dim} C_{B} \leq 1$. Moreover, $c$ is cancellable in $C_{B}$ and hence does not belong to a prime ideal of the zero ideal of $C_{B}$. Therefore $\operatorname{dim} C_{B}=1$ and the prime ideals of the zero ideal of $C_{B}$ are all isolated since $m$ is not among them. Thus by (c), $C_{B}$ is a basic ring.

(h) $A$ is the total quotient ring of $B$, and $3^{\circ}$ of Definition 2 holds for $B$. First, $A$ is contained in the total quotient ring of $B$, for if $z \in A$, then $c^{n} z \in B$ for some $n \geq 1$ since $\lim c^{n} z=0$, so $z=c^{-n}\left(c^{n} z\right)$ is the quotient of an element of $B$ and a cancellable element of $C_{B}$. We need only prove, therefore, that a cancellable element $d$ of $C_{B}$ is invertible in $C$ (and hence invertible in $A$ and cancellable in $B$ ). We may assume that $d$ is not invertible in $C_{B}$, i.e., that $d \in \mathrm{m}$. By $(\mathrm{g}), \mathrm{m}$ is the only prime ideal of $C_{B}$ containing $d$, so $d C_{B}$ is primary for $m$ and hence is open in $C_{B}$ and therefore also in $C$. The ideal $d C$ of $C$ generated by $d C_{B}$ is consequently open in $C$, so $d C=C$ by hypothesis, whence $d$ is invertible in $C$. To complete the proof of the theorem, therefore, we need only prove that $2^{\circ}$ of Definition 2 holds for $B$. 
(i) The topology of $B$ is its natural topology as a $C_{B}$-module. First we shall show that $\mathfrak{m}^{n} B$ is open. $B y(c)$ and Definition 1 , there is an open left ideal $a$ of $B$ such that $a \cap C_{B} \subseteq m^{n}$. Then as $a \cap C_{B}$ is open in $C,\left(a \cap C_{B}\right) C$ is an open ideal of $C$, so $\left(a \cap C_{B}\right) C=C$, and consequently there exist $a_{1}, \ldots, a_{s} \in$ $a \cap C_{B}, c_{1}, \ldots, c_{s} \in C$ such that $a_{1} c_{1}+\cdots+a_{s} c_{s}=1$. Now $b=\{x \in B$ : $\left.c_{i} x \in B, 1 \leq i \leq s\right\}$ is an open right ideal of $B$ contained in $\mathfrak{m}^{n} B$, for if $x \in \mathcal{D}$, then $x=a_{1}\left(c_{1} x\right)+\cdots+a_{s}\left(c_{s} x\right) \in\left(a \cap C_{B}\right) B \subseteq \mathfrak{m}^{n} B$. Therefore $\mathfrak{m}^{n} B$ is open. On the other hand, if $D$ is an open left ideal of $B$, then $D \cap C_{B} \supseteq \mathrm{m}^{k}$ for some $k \geq 1$ by (c), so $\mathrm{m}^{k} B=B \mathrm{~m}^{k} \subseteq B D=0$. Therefore $\left(\mathrm{m}^{n} \cdot B\right)_{n \geq 1}$ is a fundamental system of neighborhoods of zero.

(j) $B$ is a noetherian $C_{B}$-module. By (i), $\bigcap_{n=1}^{\infty} \overline{m^{n} B}=(0)$. Therefore by (a), (b), (i), and [15, Theorem 11], $m B$ is a finitely generated $C_{B}$-submodule. Also as $B$ is a strictly linearly compact $C_{B}$-module, $B / \mathrm{m} B$ is an artinian $C_{B}$ module by (i); regarded as a vector space over $C_{B} / \mathrm{m}$, therefore, $B / \mathrm{m} B$ is artinian, thus finite-dimensional, and hence noetherian. Consequently, $B / \mathrm{m} B$ is a noetherian $C_{B}$-module. $A s \mathrm{~m} B$ is a finitely generated module over $C_{B}$, a noetherian ring, we conclude that $\mathrm{m} B$ is also a noetherian $C_{B}$-module. Consequently, $B$ itself is a noetherian $C_{B}$-module.

Lemma 4. If $A$ is a locally centrally linearly compact ring with identity and if the center $C$ of $A$ is a topological ring baving no proper open ideals, then there is an open, centrally linearly compact subring $B$ of $A$ that contains 1.

Proof. By hypothesis, $A$ contains an open, centrally linearly compact subring $B^{\prime}$. Let $B$ be the subring of $A$ generated by $B^{\prime}$ and 1. Every left ideal $a$ of $B^{\prime}$ is also a left ideal of $B$, for $V_{a}=\{b \in A: b a \subseteq a\}$ is a subring of $A$ that contains $B^{\prime}$ and 1 and hence contains $B$. Thus every open left ideal of $B^{\prime}$ is an open left ideal of $B$, so the open left ideals of $B$ form a fundamental system of neighborhoods of zero. By hypothesis, the ideal of $C$ generated by $B^{\prime} \cap C$ is $C$, i.e., $C=\left(B^{\prime} \cap C\right) C$. In particular, there exist $x_{1}, \ldots, x_{n} \in B^{\prime} \cap C$ and $c_{1}, \ldots, c_{n} \in C$ such that $1=x_{1} c_{1}+\cdots+x_{n} c_{n}$. Since $B \subseteq V_{B^{\prime}}$, for any $b \in B$ we have $b=\left(b x_{1}\right) c_{1}+$ $\cdots+\left(b x_{n}\right) c_{n} \in B^{\prime} c_{1}+\cdots+B^{\prime} c_{n}$. Since $B^{\prime}$ is a strictly linearly compact module over its center $C_{B^{\prime}}, B^{\prime} c_{1}+\cdots+B^{\prime} c_{n}$ is also a strictly linearly compact $C_{B^{\prime}}$ module, for it is continuous homomorphic image of the $C_{B^{\prime}}$-module $B^{\prime n}$. Consequently, as $B$ is an open and thus closed $C_{B^{\prime}}$-submodule of $B^{\prime} c_{1}+\cdots+B^{\prime} c_{n}$, $B$ is strictly linear compact over $C_{B^{\prime}}$. As the open left ideals of $B$ form a fundamental system of neighborhoods of zero, therefore, $B$ is also strictly linearly compact over its center. Thus $B$ is an open, centrally linearly compact subring that contains 1 .

We recall that if $\left(A_{\gamma}\right)_{\gamma \in \Gamma}$ is a family of topological rings and if, for each 


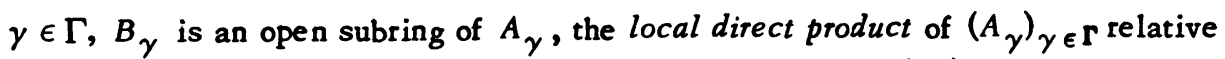
to $\left(B_{\gamma}\right)_{\gamma \in \Gamma}$ is the subring $D$ of $\Pi_{\gamma \in \Gamma} A_{\gamma}$ consisting of all $\left(x_{\gamma}\right)$ such that $x_{\gamma} \in$ $B_{\gamma}$ for all but finitely many $\gamma \in \Gamma$, equipped with the topology obtained by declaring the open neighborhoods of zero in $\Pi_{\gamma \in \Gamma} \Gamma_{\gamma}$ (equipped with the cartesian product topology) a fundamental system of neighborhoods of zero in $D$. It is easy to verify that $D$ is a topological ring, that the canonical injection from $A_{\gamma}$ into $D$ is a topological isomorphism from $A_{\gamma}$ onto an ideal of $D$, and that the projection from $D$ onto $A_{\gamma}$ is a continuous open epimorphism. It is also apparent that

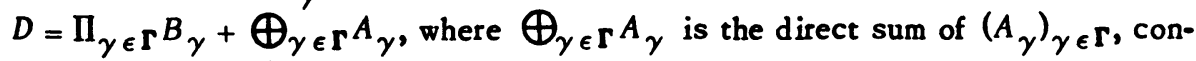
sisting of all $\left(x_{\gamma}\right)$ such that $x_{\gamma}=0$ for all but finitely many $\gamma \in \Gamma$.

Our structure theorem for a locally centrally linearly compact ring with identity whose center is a topological ring without proper open ideals is the following:

Theorem 10. Let $A$ be a Hausdorff topological ring with identity. The following statements are equivalent:

1. $A$ is locally centrally linearly compact, and the center $C$ of $A$ is a topological ring baving no proper open ideals.

20. $A$ is topologically isomorpbic to the local direct product of $\left(A_{\gamma}\right)_{\gamma \in \mathbf{r}}$

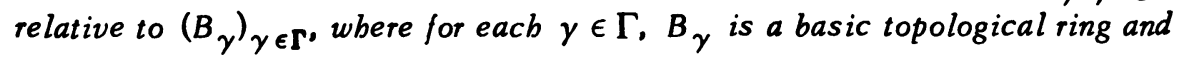
$A_{\gamma}$ is the topological quotient ring of $B_{\gamma}$.

Moreover, if the additive order of each element of $A$ is either infinite or squarefree, then each $A_{\gamma}$ is the topological direct sum of finitely many ideals, each a finite-dimensional Hausd orff topological algebra with identity over a local field whose center is a Coben subalgebra.

Proof. Assume $1^{\circ}$. By Lemma 4, there is an open, centrally linearly compact subring $B$ of $A$ such that $1 \in B$. By Lemma 3, the center of $B$ is $B \cap C$. Thus $B \cap C$ is a strictly linearly compact commutative ring with identity, so $B \cap C$ is topologically is omorphic to the cartesian product of strictly linearly compact local rings [4, Exercise 21(d), p. 112]. Thus $B \cap C$ contains a summable orthogonal family $\left(e_{\gamma}\right)_{\gamma \in \Gamma}$ of idempotents such that $\Sigma_{\gamma \in \Gamma^{e}}{ }_{\gamma}=1$ and for each $\gamma \in \Gamma$, $(B \cap C) e_{\gamma}$ is a local, strictly linearly compact ring. Clearly $\mathrm{Ce}_{\gamma}$ is the center of the ring $A e_{\gamma}, B e_{\gamma}=B \cap A e_{\gamma}$ and hence $B e_{\gamma}$ is open in $A e_{\gamma}$, and $B e_{\gamma} \cap$ $\mathrm{Ce}_{\gamma}$ is the local ring $(B \cap C) e_{\gamma} \cdot A s x \mapsto x e_{\gamma}$ is a continuous epimorphism from $C$ onto $C e_{\gamma}$, the topological ring $C e_{\gamma}$ has no proper open ideals. Therefore by Lemma 3, the center of $B e_{\gamma}$ is the local ring $B e_{\gamma} \cap C e_{\gamma}=(B \cap C) e_{\gamma}$. Since the intersection of an open left ideal of $B$ with $B e_{\gamma}$ is an open left ideal of $B e_{\gamma}$, the open left ideals of $B e_{\gamma}$ form a fundamental system of neighborhoods of zero for the induced topology of $B e y$. Since the $(B \cap C)$-module $B e_{y}$ is a continuous homomorphic image of the $(B \cap C)$-module $B$, the former module is 
strictly linearly compact; however, as $e_{\gamma} \in C$, the $(B \cap C)$-submodules of $B e_{\gamma}$ coincide with the $(B \cap C) e_{\gamma}$-submodules of $B e_{\gamma}$; consequently, the $(B \cap C) e_{\gamma}$ module $B e_{\gamma}$, with its induced topology, is strictly linearly compact. Therefore $B e_{\gamma}$ is an open, centrally linearly compact subring of $A e_{\gamma} \cdot$ By Theorem 9, Be $\gamma$ is a basic topological ring, and $A e_{\gamma}$ is the topological quotient ring of $B e \gamma^{\bullet}$

We shall next establish that $F: x \mapsto\left(x e_{\gamma}\right)_{y \in \Gamma} \in \Pi_{\gamma \in \Gamma}\left(A e_{\gamma}\right)$ is a topological isomorphism from $A$ onto the local direct product $D$ of $\left(A e_{\gamma}\right)_{\gamma \in \Gamma}$ relative to $\left(B e_{\gamma}\right)_{\gamma \in \Gamma \text { If }} a \epsilon$ $\Gamma$ and if $x \in A e_{a}$, then $F(x)=\left(x_{\gamma}\right)$, where $x_{a}=x$ and $x_{\gamma}=0$ if $\gamma \neq \alpha$. Thus $F(A) \supseteq \bigoplus_{\gamma \in \Gamma^{A e}}$. For the same reason, $\left.\bigoplus_{\gamma \in \Gamma^{B e}} \subseteq F(B) \subseteq \Pi_{\gamma \in \Gamma^{(B e}}\right)$. Since $F$ is clearly continuous, $F(B)$ is a linearly compact $(B \cap C)$-module and hence is complete; as $\bigoplus_{\gamma \in \Gamma} B e_{\gamma}$ is dense in $\Pi_{\gamma \in \Gamma}\left(B e_{\gamma}\right)$, therefore, $F(B)=$ $\Pi_{\gamma \in \Gamma}\left(B e_{\gamma}\right)$. Consequently, $F(A) \supseteq \Pi_{\gamma \in \Gamma}\left(B e_{\gamma}\right)+\bigoplus_{\gamma \in \Gamma} A e_{\gamma}=D$. For each $x \in A$,

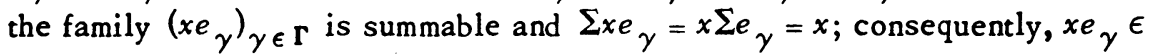
$B \cap A e_{\gamma}=B e_{\gamma}$ for all but finitely many $\gamma \in \Gamma$; therefore $F(x) \in D$. Consequently, $F(A)=D$. Moreover, $F$ is an isomorphism from $A$ on to $D$, for if $F(x)=0$, then $x e_{y}=0$ for all $y \in \Gamma$, so $x=x \Sigma e_{y}=\Sigma x e_{y}=0$. Since the restriction $F_{B}$ of $F$ to $B$ is a continuous isomorphism from the $(B \cap C)$-module $B$ onto the $(B \cap C)$ module $\Pi_{\gamma \in \Gamma}\left(B e_{\gamma}\right)$, and since the $(B \cap C)$-module $B$ is strictly linearly compact, $F_{B}$ is a topological isomorphism by the definition of a strictly linearly compact module. Consequently by the definition of the topology of $D, F$ is a topological isomorphism from $A$ to $D$.

Assume $2^{\circ}$, and let $D$ be the local direct product of $\left(A_{\gamma}\right)_{\gamma \in \Gamma \text { relative to }}$ $\left(B_{\gamma}\right)_{\gamma \in \Gamma^{*}}$ Let $B=\Pi_{\gamma \in \Gamma^{B}}$, and let $C_{\gamma}$ be the center of $A_{\gamma}$. Clearly the center $C$ of $D$ is $\left(\mathrm{II}_{\gamma \in \mathrm{I}} C_{\gamma}\right) \cap D$, so $B \cap C=\Pi_{\gamma \in \mathrm{\Gamma}}\left(B_{\gamma} \cap C_{\gamma}\right)$. By $6^{\circ}$ of Theorem 3, each $C_{\gamma}$ is a topological ring having no proper open ideals. Let $D$ be an open ideal of $C$. For each $\gamma \in \Gamma$, the projection $D_{\gamma}$ of $D$ on $A_{\gamma}$ is an open ideal of $C_{\gamma}$, so $D_{\gamma}=C_{\gamma}$. Since $C=\left(\Pi_{\gamma \in \Gamma} C_{\gamma}\right) \cap D$, therefore, an easy argument establishes that $D=C$. Consequently, $C$ is a topological ring having no proper open ideals. By Lemma 3, the center of each $B_{\gamma}$ is $B_{\gamma} \cap C_{\gamma}$, and the center of $B$ is $B \cap C$. For each $\alpha \in \Gamma, B_{\alpha}$ admits the structure of a $(B \cap C)$-module under the scalar multiplication $\left(x_{\gamma}\right) \cdot y=x_{\alpha} y$, and the $(B \cap C)$-submodules of $B_{a}$ are identical with the $\left(B_{\alpha} \cap C_{\alpha}\right)$-submodules of $B_{a}$. Therefore as $B_{a}$ is a strictly linearly compact $\left(B_{\alpha} \cap C_{\alpha}\right)$-module, it is also a strictly linearly compact $(B \cap C)$ module. Hence $B$ is a strictly linearly compact $(B \cap C)$-module [11, Theorem 5$]$. Since the open left ideals of $B_{\gamma}$ form a fundamental system of neighborhoods of zero in $B_{\gamma}$ for each $\gamma \in \Gamma$, the open left ideals of $B$ clearly form a fundamental system of neighborhoods of zero in $B$. Thus $D$ is locally centrally linearly compact, and $1^{\circ}$ holds.

We wish to extend Theorem 10 to allow for a connected factor. For this, we need the following three lemmas: 
Lemma 5. If $E$ is a locally compact, connected, unitary left [right] topological module over a locally centrally linearly compact ring $A$ with identity whose center is a topological ring baving no proper open ideals, then $E=(0)$.

Proof. By Theorem 10, there are an orthogonal summable family $\left(e_{\gamma}\right)_{\gamma \in \Gamma \text { of }}$ central idempotents and an open subring $B$ containing 1 such that $\Sigma e_{\gamma}=1$, $B e_{\gamma}$ is a basic ring, and $A e_{\gamma}$ is the topological quotient ring of $B e_{\gamma}$. Assume that $E$ is a nonzero left topological $A$-module. If $x$ is a nonzero element of $E$, then $x=1 \cdot x=\Sigma\left(e_{y} \cdot x\right)$, so there exists $\alpha \in \Gamma$ such that $e_{a} \cdot E \neq(0)$. Decomposing $A e_{a}$ in accordance with Theorem 7, we may assume further that $B e_{a}$ is a special basic ring. Now $e_{a} E$ is connected since it is a continuous image of $E ; e_{a^{\cdot}} E$ is also closed in $E$ and hence is locally compact, for if $\mathcal{F}$ is a filter on $e_{a} \cdot E$ converging to $x \in E$, then $e_{a} \mathcal{F}$ converges to $e_{a} \cdot x$, but $e_{a} \cdot \mathcal{F}=\mathcal{F}$, whence $x=e_{a} \cdot x \in e_{a} \cdot E$. Consequently, $M=e_{a} \cdot E$ is a nonzero, connected, locally compact, unitary topological module over the center $C_{a}$ of $A e_{a}$, and $C_{a}$ is a local artinian ring (or equivalently, a noetherian primary ring) by $2^{\circ}$ of Theorem 3. Let $\mathrm{m}$ be the maximal ideal of $C_{a}$. By Theorem $4, C_{\alpha} / \mathrm{m}$ is a local field. As $m$ is nilpotent, there is a smallest natural number $r$ such that $\overline{\mathfrak{m}^{\gamma} \cdot M}$ is properly contained in $M$. Thus $M_{r}=M / \overline{m^{\top} \cdot M}$ is a nonzero, locally compact, connected topological vector space over the local field $C / m$, for by continuity, $\mathfrak{m} \cdot M=\mathfrak{m} \cdot \overline{\mathfrak{m}^{r-1} \cdot M} \subseteq \overline{\mathfrak{m}^{r} \cdot M}$. Consequently, $M_{r}$ is finite dimensional over $C / \mathrm{m}[3$, Theorem $3, \mathrm{p} .20]$, so the connected space $M_{r}$ is topologically isomorphic to the totally disconnected space $(C / m)^{s}$ for some $s>0[3$, Theorem 2 , p. 18], a contradiction.

We are indebted to John S. Cook for a discussion concerning the proof of the following lemma:

Lemma 6. Let $A$ be a Hausdorff topological ring, and let $c$ be the connected component of zero. If $A$ bas a left [right] identity, if $C$ is locally compact and not the zero ideal, and if $A / C$ is a locally centrally linearly compact ring with identity whose center is a topological ring baving no proper open ideals, then $c$ is a finite-dimensional topological algebra over $\mathbf{R}$, the right [left] annibilator of $\mathrm{C}$ in $\mathrm{C}$ is (0), and c contains a nonzero idempotent.

Proof. We assume that $A$ has a left identity element $e$; let $\phi$ be the canonical epimorphism from $A$ onto $A / c$. By the Jacobson-Taussky theorem [13, Theorem 5], $C$ contains a connected, compact ideal $G$ such that $c h=(0)$ and $\mathrm{c} / \mathrm{G}$ is a finite-dimensional topological algebra over $\mathbf{R}$. Since $A / c$ is a ring with identity, its identity element is $\phi(e)$. Therefore as $c h=(0), 5$ is a topological unitary left module over $A / c$. By Lemma $5, \bar{G}=(0)$; therefore $c$ is a finitedimensional topological algebra over R. Let $a=\{x \in c: c x=(0)\}$. Clearly $a$ 
is a closed ideal of $A$; also $a$ is clearly a subspace of the R-algebra $c$ and hence is connected. Therefore as $c a=(0), a$ is a topological unitary left module over $A / c$, so $a=(0)$ by Lemma 5 .

Consequently, the right regular representation $R: c \mapsto R_{c}$ from $c$ into End ${ }_{R}(c)$, where $R_{c}$ is defined by $R_{c}(x)=x c$ for all $x \in c$, is an antimonomorphism. As $c$ is a finite-dimensional algebra, its radical is nilpotent, so $c$ is not a radical ring as $a=(0)$, and consequently $c$ contains a nonnilpotent element $u$. The sequence $\left(R_{u}^{n}(c)\right)_{n \geq 1}$ of subspaces of $c$ is decreasing, and hence for some $m, R_{u m}(\mathrm{c})=R_{u}^{m}(\mathrm{c})=R_{u}^{s}(\mathrm{c})=R_{u} s(\mathrm{c})$ for all $s \geq m$. Let $v=u^{m}$, and let $M=R_{v}(\mathrm{c})$. Then $M$ is the range of $R_{v}^{u}, M \neq(0)$ since $u$ is not nilpotent, and $R_{v}(M)=R_{u}{ }^{2 m}(\mathrm{c})=M$. Consequently, the restriction $v_{M}$ of $R_{v}$ to $M$ is an automorphism of the R-vector space $M$. Therefore, the characteristic polynomial $X^{n}+\cdots+a_{1} X+a_{0}$ of $v_{M}$ has a nonzero constant term $a_{0}$. Let $p=$ $-\alpha_{0}^{-1}\left(v^{n}+\cdots+\alpha_{1} v\right)$. Then $p \in c, R_{p}(c) \subseteq M$, and by the Cayley-Hamilton theorem, the restriction of $R_{p}$ to $M$ is the identity linear operator. Hence $R_{p}$ is a projection on $M$, so as $R$ is an antimonomorphism, $p$ is a nonzero idempotent.

Lemma 7. Let $A$ and $A^{\prime}$ be topological rings with identity elements 1 and $1^{\prime}$, and let $\phi$ be a continuous bomomorphism from $A$ into $A^{\prime}$ such that $\phi(1)=1^{\prime}$. If $C$ is a subring of $A$ containing 1 such that the topological ring $C$ contains no proper open ideals and if $C^{\prime}$ is a subring of $A^{\prime}$ containing $\phi(C)$, then the topological ring $C^{\prime}$ contains no proper open ideals.

Proof. Let $D^{\prime}$ be an open ideal of $C^{\prime}$. There exists an open set $U^{\prime}$ in $A^{\prime}$ such that $U^{\prime} \cap C^{\prime}=D^{\prime}$. Moreover, $\phi^{-1}\left(U^{\prime}\right) \cap C$ is an (open) ideal of $C$; indeed, if $x \in C$ and $y \in \phi^{-1}\left(U^{\prime}\right) \cap C$, then $\phi(x) \in \phi(C) \subseteq C^{\prime}$ and $\phi(y) \in \phi\left(\phi^{-1}\left(U^{\prime}\right) \cap C\right)$ $\subseteq U^{\prime} \cap C^{\prime}=D^{\prime}$, so $\phi(x y)=\phi(x) \phi(y) \in C^{\prime} D^{\prime}=D^{\prime}$, whence $x y \in \phi^{-1}\left(U^{\prime} \cap C^{\prime}\right) \cap C \subseteq$ $\bar{\phi}^{-1}\left(U^{\prime}\right) \cap C$; similarly, if $x, y \in \phi^{-1}\left(U^{\prime}\right) \cap C$, then $x-y \in \phi^{-1}\left(U^{\prime}\right) \cap C$. Consequently, $\phi^{-1}\left(U^{\prime}\right) \cap C=C$, so $1 \in C \subseteq \phi^{-1}\left(U^{\prime}\right)$, whence $1^{\prime}=\phi(1) \in U^{\prime} \cap C^{\prime}=$ $D^{\prime}$, and therefore $D^{\prime}=C^{\prime}$.

For convenience, let us call a topological ring $A$ an $S$-ring if $A$ is a Hausdorff topological ring with identity, if the connected component $c$ of zero in $A$ is locally compact, and if either $A=c$ or $A / C$ is locally centrally linearly compact. Thus the topological rings satisfying $(a)-(c)$ of $\$ 1$ are precisely the $S$-rings whose centers are topological rings having no proper open ideals. Here is our structure theorem for such rings:

Theorem 11. Let $A$ be a Hausdorff topological ring with identity, and let $c$ be the connected component of zero in $A$. The following statements are equivalent: 
1. $A$ is an S-ring, and the center of $A$ is a topological ring baving no proper open ideals.

2. $A$ is an S-ring, and the center of $A / C$ is a topological ring baving no proper open ideals.

30. $A$ is topologically isomorphic to $A_{1} \times \cdots \times A_{n} \times D(n \geq 0)$, where each $A_{i}$ is a finite-dimensional Hausdorff topological algebra with identity over $\mathbf{R}$ or C whose center is a Coben subalgebra, and where either $D=(0)$ or $D$ is the local

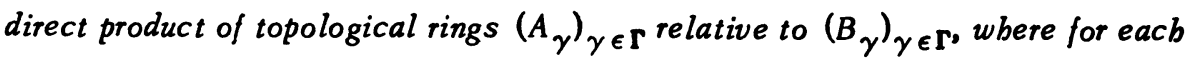
$\gamma \in \Gamma, B_{\gamma}$ is a basic topological ring and $A_{\gamma}$ is the topological quotient ring of $\boldsymbol{B}^{*}$

Moreover, the final statement of Theorem 10 pertains.

Proof. Assuming that $c$ is a proper ideal of $A$ and applying Lemma 7 to the centers $C$ and $C^{\prime}$ of $A$ and $A / C$ respectively and to the canonical epimorphism $\phi$ from $A$ onto $A / c$, we conclude that $1^{\circ}$ implies $2^{\circ}$.

To show that $2^{\circ}$ implies $3^{\circ}$, we may assume by Theorem 10 , that $c \neq(0)$. We shall first prove that $c$ contains an identity element. The assertion holds by hypothesis if $A=c$, so we shall assume that $A / c \neq(0)$. By Lemma $6, c$ is a finite-dimensional Hausdorff topological algebra over $\mathbf{R}$ and the left annihilator of $C$ in $C$ is (0); therefore the left regular representation $L: c \mapsto L_{c}$ from $c$ into End $_{R}(c)$, where $L_{c}$ is defined by $L_{c}(x)=c x$ for all $x \in c$, is a monomorphism. Let $e$ be an idempotent in $c$ such that $L_{e}(c)$ is maximal in the set of all the subspaces $L_{p}(c)$ where $p$ is an idempotent of $c$.

Suppose that $(1-e) c \neq(0)$. Let $\phi^{\prime}$ be the restriction of $\phi$ to $(1-e) A$, and let $\pi$ be the continuous open epimorphism $x \mapsto(1-e) x$ from the additive topological group $A$ onto the additive topological group $(1-e) A$. Since $e \in \mathrm{C}, \phi=$ $\phi^{\prime} \pi$; therefore as both $\phi$ and $\pi$ are continuous and open, $\phi^{\prime}$ is a continuous open epimorphism from $(1-e) A$ onto $A / c$. The kernel of $\phi^{\prime}$ is $(1-e) A \cap c=(1-e) c$; therefore $(1-e) A /(1-e) C$ is topologically isomorphic to $A / C$. Clearly $(1-e) c$ is closed in $c$ and hence is locally compact; $(1-e) c$ is the continuous image of a connected set and hence is connected; as the connected component of zero in $(1-e) A$ is clearly contained in $(1-e) A \cap c$, therefore, we conclude that $(1-e) c$ is the connected component of zero in $(1-e) A$. By Lemma 6 applied to $(1-e) A$, which has the left identity $1-e,(1-e) c$ has a nonzero idempotent $f$. As $f=$ $(1-e) f$, ef $=0$. Let $M=L_{e}(c), N=L_{f}(c)$, and let $g=e+f$. Then $L_{g}(\mathrm{c}) \subseteq$ $M+N$; however, as ef $=0, L_{\boldsymbol{g}}\left(m-L_{f}(m)\right)=m$ for each $m \in M$, and $L_{\boldsymbol{g}}(n)=\bar{n}$ for each $n \in N$. The restriction of $L_{g}$ to $M+N$ is therefore an automorphism of the R-vector space $M+N$. Applying the Cayley-Hamilton theorem as in the proof of Lemma 6 , we conclude that there is an idempotent $b$ in $c$ such that $L_{b}$ is 
a projection on $M+N$. Since $M \cap N=(0)$ (as $e f=0$ ) and since $N \neq(0)$, we thus obtain a contradiction.

Therefore $(1-e) c=(0)$, so $L_{e}$ is the identity linear operator on $c$, and consequently $e$ is the identity element of $c$. Moreover, $e$ is a central idempotent of $A$, for if $x \in A$, then $e x$ and $x e$ belong to $c$, so $e x=(e x) e=e(x e)=x e$. Consequently, $A$ is the topological direct sum of the ideals $A e=c$ and $A(1-e)$, which is topologically isomorphic to $A / C$. Therefore $3^{\circ}$ holds by Theorem 10 and a remark of $\$ 1$ concerning finite-dimensional algebras with identity (for if $A$ is a finite-dimensional local R-algebra, by the proof of Cohen's theorem [6, Theorem 9], $\mathbf{R} \cdot \mathbf{1}$ is contained in a coefficient field that is a finite-dimensional topological Ralgebra and hence is topologically isomorphic to $\mathbf{R}$ or $\mathbf{C}$ ).

Since the center of a cartesian product of rings is the cartesian product of their centers, it is easy to see that $3^{\circ}$ implies $1^{\circ}$ by Theorem 10.

To apply Theorem 11 to locally compact rings, we need the following lemma:

Lemma 8. Let $A$ be the topological quotient ring of a basic ring $B$. The following statements are equivalent:

1. $A$ is locally compact.

$2^{\circ}$. $B$ is compact.

30. The residue field of the center of $B$ is finite.

Furthermore, if $B$ is special, the following statement is equivalent to $1^{\circ}-3^{\circ}$ :

4. The residue field of the center $C$ of $A$ is a locally compact field.

Proof. Let $m$ be the maximal ideal of the center $C_{B}$ of $B$. If $B$ is compact, then the residue field $C_{B} / \mathrm{m}$ of $C_{B}$ is compact and discrete and hence is finite. Conversely, assume that $C_{B} / \mathrm{m}$ is finite. Then for each $n \geq 1, \mathrm{~m}^{n} / \mathrm{m}^{n+1}$ is finite since it is a finite-dimensional $\left(C_{B} / \mathrm{m}\right)$-vector space. By induction, $C_{B} / \mathrm{m}^{\tau}$ is finite for all $r \geq 1$; hence $C_{B}$ is precompact. As $C_{B}$ is complete, therefore, $C_{B}$ is compact. Thus $2^{\circ}$ and $3^{\circ}$ are equivalent.

Clearly $2^{\circ}$ implies $1^{\circ}$. If $1^{\circ}$ holds, then $m^{n} B$ is compact for some $n \geq 1$, so $\mathfrak{m}^{n} B / \mathfrak{m}^{n+1} B$ is compact and discrete and hence is finite. But since $B$ is not discrete, $\mathfrak{m}^{n} B / \mathrm{m}^{n+1} B$ is a nonzero vector space over $C_{B} / \mathrm{m}$, so $C_{B} / \mathrm{m}$ is finite. Thus $1^{\circ}$ implies $3^{\circ}$.

Finally, assume that $B$ is special. Clearly $1^{\circ}$ implies $4^{\circ}$. Assume $4^{\circ}$, and let $\phi$ be the canonical epimorphism from $C$ onto its residue field $K$, a local field by Theorem 4. By Lemma $2, \phi\left(C_{B}\right)$ is contained in the valuation ring $V$ of $K$, which is compact by hypothesis. Let $M$ be the maximal ideal of $V$, and let $n=\phi^{-1}(\mathbb{R}) \cap C_{B}$. Then $C_{B} / n$ is isomorphic to a subring of $V / \mathbb{N}$, a finite field, so $C_{B} / n$ is a finite field; hence $n=m$ and $3^{\circ}$ holds.

Theorem 12. Let $A$ be a Hausdorff topological ring with identity. The following statements are equivalent: 
10. A is a locally compact ring whose center is a topological ring baving no proper open ideals.

20. A is topologically isomorphic to $A_{1} \times \cdots \times A_{n} \times D(n \geq 0)$, where each $A_{i}$ is a finite-dimensional Hausdorff topological algebra over $\mathbf{R}$ or $\mathbf{C}$ whose center is a Coben subalgebra, and where either $D=(0)$ or $D$ is the local direct product

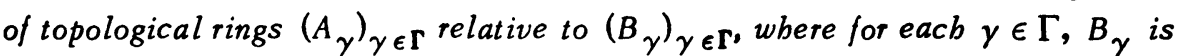
a compact basic ring and $A_{\gamma}$ is the topological quotient ring of $B_{\gamma}$.

Moreover, if the additive order of each element of $A$ is either infinite or squarefree, then each $A_{\gamma}$ is the topological direct sum of finitely many ideals, each a finite-dimensional locally compact algebra with identity over a totally disconnected, indiscrete, locally compact field.

Proof. If $A$ is locally compact and satisfies $3^{\circ}$ of Theorem 11 then each $A_{\gamma}$ is the image of $A$ under a continuous open homomorphism and hence is locally compact, so each $B_{\gamma}$ is compact by Lemma 8 . Thus $1^{\circ}$ implies $2^{\circ}$. Conversely, if each $B_{\gamma}$ is compact, then $D$ is clearly locally compact by Tihonov's theorem, so $A$ is also locally compact. By Theorem 11 , therefore, $1^{\circ}$ and $2^{\circ}$ are equivalent; the final statement follows from Theorem 11 and Lemma 8.

Theorem 13. Let $A$ be a Hausdorff topological ring with identity, and let $C$ be the center of $A$. The following statements are equivalent:

$1^{\circ}$. $A$ is an S-ring, and $C$ is a topological ring baving no proper open ideals or nonzero nilpotents.

$2^{\circ}$. A is topologically isomorphic to $A_{1} \times \cdots \times A_{n} \times D(n \geq 0)$, where each $A_{i}$ is a central, finite-dimensional Hausdorff topological algebra over $\mathbf{R}$ or $\mathbf{C}$, and where either $D=(0)$ or $D$ is the local direct product of topological rings

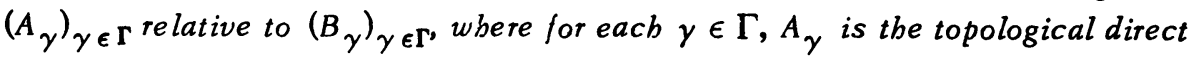
sum of finitely many ideals, each a central, finite-dimensional Hausdorff topological algebra over a local field, and where $B_{y}$ is a basic ring of which $A_{y}$ is the topological quotient ring.

Proof. A commutative local artinian ring without nonzero nilpotents is a field. In particular, a finite-dimensional local R-algebra without nonzero nilpotents is a finite-dimensional field extension of $\mathbf{R}$ and hence is $\mathbf{R}$ or $\mathbf{C}$; and if the center of the topological quotient ring of a special basic ring has no nonzero nilpotents, then it is a local field by $2^{\circ}$ of Theorem 3 and Theorem 4 . The equivalence of $1^{\circ}$ and $2^{\circ}$ therefore follows from Theorems 11 and 7.

Theorem 14. Let $A$ be a Hausdorff topological ring with identity, and let $C$ be the center of $A$. The following statements are equivalent: 
1. $A$ is an S-ring that has no nonzero nilpotent ideals, and the topological ring $C$ bas no proper open ideals.

20. $A$ is topologically isomorpbic to $A_{1} \times \cdots \times A_{n} \times D(n \geq 0)$, where eacb $A_{i}$ is the topological ring of all square matrices of some order over $\mathrm{R}, \mathrm{C}$, or the division ring $\mathbf{H}$ of quaternions, and where either $D=(0)$ or $D$ is the local direct

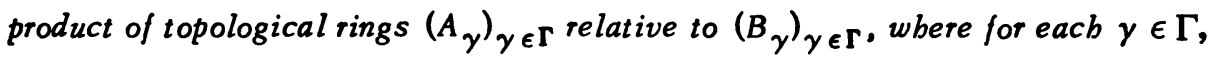
$A_{\gamma}$ is the topological direct sum of finitely many ideals, each the topological ring of all square matrices of some order over a division ring finite dimensional over its center, which is a local field, and where $B_{\gamma}$ is a basic ring of wbich $A_{\gamma}$ is the topological quotient ring.

Proof. Statements $1^{\circ}$ and $2^{\circ}$ imply the corresponding statements of Theorem 13. We observe that, with the notation of Theorem 13, $A$ has no nonzero nilpotent ideals if and only if each $A_{i}$ and each $A_{\gamma}$ have no nonzero nilpotent ideals, or equivalently, by $3^{\circ}$ of Theorem 3 , are semisimple. In particular, $2^{\circ}$ implies $1^{\circ}$. On the other hand, $1^{\circ}$ implies $2^{\circ}$ by Theorem 13 and Wedderburn's theorem on finitedimensional semisimple algebras.

Specializing either Theorem 12 or Theorem 13 to the commutative, locally compact, semisimple case, we obtain the structure theorem of Goldman and Sah [7, Theorem 4.1].

Theorem 15. Let $A$ be a Hausdorff topological ring with identity, and let $C$ be the center of $A$. The following statements are equivalent:

1. $A$ is an S-ring, $C$ is a topological ring that has no proper open ideals, and any one and bence all of the following conditions bold:

(a) Contains an invertible element $c$ sucb that $\lim c^{n}=0$.

(b) $A$ bas only finitely many maximal ideals.

(c) Every ideal [left ideal, right ideal] of $A$ is closed.

(d) $A$ is a (left or right) noetherian [artinian] ring.

2. $A$ is topologically isomorpbic to $A_{1} \times \cdots \times A_{n}$, where each $A_{i}$ is either a finite-dimensional Hausdorff topological algebra over $\mathbf{R}$ or $\mathbf{C}$ whose center is a Coben subalgebra or the topological quotient ring of a special basic ring.

Proof. To show that $1^{\circ}$ implies $2^{\circ}$, we may assume by Theorem 11 that $A$ is

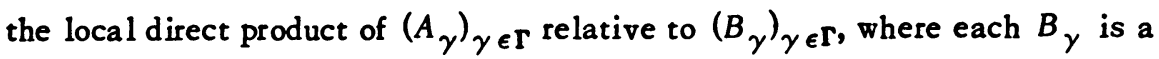
basic ring and $A_{\gamma}$ is the topological quotient ring of $B_{\gamma}$. By Theorem 7, it suffices to show that $\Gamma$ is finite. Assume first that $c=\left(c_{\gamma}\right)$ is an invertible element of $C$ such that $\lim c^{n}=0$. Replacing $c$ with a power of $c$, if necessary, we may assume that $c \in\left(\Pi_{\gamma \in \Gamma} B_{\gamma}\right) \cap C=\Pi_{\gamma \in \Gamma}\left(B_{\gamma} \cap C_{\gamma}\right)$, where $C_{\gamma}$ is the center of $A_{\gamma}$. Now $\lim c_{\gamma}^{n}=0$ for each $\gamma \in \Gamma$, so $c_{\gamma}$ belongs to the maximal ideal of 
the center $B_{\gamma} \cap C_{y}$ of $B_{\gamma}\left(1^{\circ}\right.$ of Theorem 3). Thus $c_{\gamma}^{-1} \notin B$ for all $\gamma \in \Gamma$. But as $c^{-1}=\left(c_{\gamma}^{-1}\right) \in A, c_{\gamma}^{-1} \notin B_{\gamma}$ for only finitely many $\gamma \in \Gamma$, so $\Gamma$ is finite.

If $\mathbb{R}_{\beta}$ is a maximal ideal of $A_{\beta}$, then $\mathbb{M}_{\beta}^{\prime}=\left\{\left(x_{\gamma}\right) \in A: x_{\beta} \in \mathbb{R}_{\beta}\right\}$ is clearly a maximal ideal of $A$ whose projection on $A_{\beta}$ is $\mathbb{M}_{\beta}$ and whose projection on every other $A_{\gamma}$ is $A_{\gamma}$. Therefore (b) implies that $\Gamma$ is finite. Since $\bigoplus_{\gamma \in \Gamma} A_{\gamma}$ is a dense ideal of $A,(c)$ also implies that $\Gamma$ is finite.

Finally, let $\mathcal{F}(\Gamma)$ be the set of all finite subsets of $\Gamma$. For each $\Delta \in \mathcal{F}(\Gamma)$, let $A_{\Delta}=\left\{\left(x_{\gamma}\right) \in A: x_{\gamma}=0\right.$ for all $\left.\gamma \notin \Delta\right\}$ and let $A_{\Delta}^{\prime}=\left\{\left(x_{\gamma}\right) \in A: x_{\gamma}=0\right.$ for all $\gamma \in \Delta\}$. If $\Gamma$ is infinite, then the set of all $A_{\Delta}$ [respectively, $A_{\Delta}^{\prime}$ ] where $\Delta \epsilon$ $\mathcal{F}(\Gamma)$ is a family of ideals having no maximal [minimal] member. Thus (d) implies that $\Gamma$ is finite.

To show that $2^{\circ}$ implies $1^{\circ}$, by Theorem 11 we need only establish (a)-(d), and for that we may assume $n=1$. If $A$ is the topological quotient ring of a special basic ring, then (a) holds by $1^{\circ}$ and $2^{\circ}$ of Definition 2, and (b)-(d) hold by Theorem 3. If $A$ is a finite-dimensional Hausdorff topological algebra over $\mathbf{R}$ or C with identity element $e$, then clearly (c) and (d) hold, (b) holds since $A / \operatorname{Rad}(A)$ is the direct sum of simple rings by Wedderburn's theorem, and finally (a) holds, for if $0<|\lambda|<1$, then $\lambda \cdot e$ is an invertible element of $C$ such that $\lim (\lambda \cdot e)^{n}$ $=0$.

Theorem 16. Let $A$ be a Hausdorff topological ring with identity. The following statements are equivalent:

1. A is the topological direct sum of finitely many ideals, each a finitedimensional Hausdorff topological algebra over $\mathbf{R}, \mathbf{C}$, or a local field.

$2^{\circ}$. The additive order of each element of $A$ is either infinite or squarefree, $A$ is an S-ring, and the center of $A$ contains an invertible element $c$ such that $\lim c^{n}=0$.

30. A is the topological direct sum of finitely many ideals, each a finitedimensional Hausdorff topological algebra over R, C, or a local field, the center of which is a Coben subalgebra.

Proof. If $c$ is an invertible element of the center $C$ such that $\lim c^{n}=0$, then any open ideal of $C$ contains the invertible element $c^{n}$ for some $n \geq 1$ and hence is $C$. Thus $1^{\circ}$ implies $2^{\circ}$ and $2^{\circ}$ implies $3^{\circ}$ by Theorems 15 and 6 .

By Theorem 15, we may replace "the center of $A$ contains an invertible element $c$ such that $\lim c^{n}=0$ " by the conjuction of "the center of $A$ is a topological ring that has no proper open ideals" and any one of (b), (c), (d) of Theorem 15.

In view of Theorem 12, specializing Theorem 16 to the locally compact case yields [13, Theorem 8$]$. 


\section{BIBLIOGRAPHY}

1. N. Bourbaki, Éléments de mathématique. Part. 1. Les structures fondamentales de l'analyse. Livre III: Topologie générale. Chaps. III, IV, 3ième éd., Actualités Sci. Indust., no. 916, Hermann, Paris, 1960. MR 41 \#984.

2. ——Eléments de mathématique. I: Les structures fondamentales de l'analyse. Fasc. VIII. Livre III: Topologie générale. Chap. 9: Utilisation des nombres réels en topologie générale, 2ième éd., Actualités Sci. Indust., no. 1045, Hermann, Paris; English transl., Hermann, Paris; Addison-Wesley, Reading, Mass., 1966. MR 30 \#3439; MR 34 \#5044b.

3. ——, Éléments de mathématique. Fasc. XV. Livre V: Espaces vectoriels topologiques. Chap. 1: Espaces vectoriels topologiques sur un corps valué. Chap. 2: Ensembles convexes et espaces localement convexes, 2ième éd., Hermann, Paris, 1966. MR 34 \#3277.

4. - Éléments de mathématique. Fasc. XXVII. Algèbre commutative. Chap. 3: Graduations, filtrations et topologies. Chap. 4: Idéaux prémiers as sociés et décomposition primaire, Actualités Sci. Indust., no. 1293, Hermann, Paris, 1961. MR 30 \#2027.

5. Eléments de mathématique. Fasc. XXX, Algèbre commutative. Chap. 5: Entiers. Chap. 6: Valuations, Actualités Sci. Indust., no. 1308, Hermann, Paris, 1964. MR $33 \# 2660$.

6. I. S. Cohen, $O_{n}$ the structure and ideal theory of complete local rings, Trans. Amer. Math. Soc. 59 (1946), 54-106. MR 7, 509.

7. Oscar Goldman and Chih-Han Sah, $O_{n}$ a special class of locally compact rings, J. Algebra 4 (1966), 71-95. MR33 \#4104.

8. Nathan Jacobson, Structure of rings, Amer. Math. Soc. Colloq. Publ., vol. 37, Amer. Math. Soc., Providence, R. I., 1956. MR 18, 373.

9. Irving Kaplansky, Topological rings, Amer. J. Math. 69 (1947), 153-183. MR 8, 434 .

10. - Locally compact rings, Amer. J. Math. 70 (1948), 447-459. MR 9, 562.

11. Horst Leptin, Linear kompakte Moduln und Ringe, Math. Z. 62 (1955), 241-267. MR 16, 1085.

12. Masayoshi Nagata, Local rings, Interscience Tracts in Pure and Appl. Math., no. 13, Interscience, New York, 1962. MR 27 \#5790.

13. Seth Warner, Locally compact rings having a topologically nilpotent unit, Trans. Amer. Math. Soc. 139 (1969), 145-154. MR 39 \#2819.

14. - Openly embedding local noetherian domains, J. Reine Angew. Math. 253 (1972), 146-151.

15. - Linearly compact rings and modules, Math. Ann. 197 (1972), 29-43.

16. - A topological characterization of complete, discretely valued fields, Pacific J. Math. 46 (1973).

17. Oscar Zariski and Pierre Samue 1, Commutative algebra. Vol. 1, University Series in Higher Math., Van Nostrand, Princeton, N. J., 1958. MR 19, 833.

18. - Commutative algebra. Vol. 2, University Series in Higher Math., Van Nostrand, Princeton, N. J., 1960. MR $22 \# 11006$.

DEPARTMENT OF MATHEMATICS, CLAREMONT MEN'S COLLEGE, CLAREMONT, CALIFORNIA 91711

DEP AR TMENT OF MATHEMATICS, DUKE UNIVERSITY, DURHAM, NORTH CAROLINA 27706 